\title{
Identification CXCL9 is a Potential Prognostic Biomarker in Ovarian and Gastric Cancer and is Correlated with Immune Infiltrates
}

Shuwei Li ( $\nabla$ yfzx_406@126.com )

Tarim University

\section{Ruijun Shi}

Tarim University

Hangzheng Zhou

Tarim University

Dongyang Wang

Tarim University

Kunlong Zhu

Tarim University

Shenglong Xue

Tarim University

Jianpei Zhang

Tarim University

Tinghui Wu

Tarim University

Yongchao Li

Tarim University

\section{Research Article}

Keywords: CXCL9, prognosis, tumor-associated macrophages, ovarian cancer, gastric cancer

Posted Date: October 1st, 2021

DOl: https://doi.org/10.21203/rs.3.rs-350037/v2

License: @ (i) This work is licensed under a Creative Commons Attribution 4.0 International License. Read Full License 


\section{Abstract}

Background: CXCL9 also known as an interferon gamma-inducible chemokine that belonging to the CXC chemokine family. It plays a role in promoting chemotaxis, inducing leukocyte differentiation and multiplication, and triggering tissue extravasation.

Methods: The TIMER (Tumor Immune Estimation Resource) and cancer microarray database Oncomine were used to dig at CXCL9 expression. The clinic prognostic level of CXCL9 was evaluated via Kaplan-Meier plotter. Then, Using TIMER and GEPIA, we investigated whether CXCL9 expression impacted cancer immune infiltrates.

Results: CXCL9 expression has been found to be significantly lower in ovarian and gastric cancers relative to normal tissues. In patients with ovarian cancer (OS HR $=0.78, \mathrm{P}=0.0017$; $\mathrm{PFS} \mathrm{HR}=0.85, \mathrm{R}=0.015)$ and gastric cancer $(\mathrm{OS} H \mathrm{H}=0.55, \mathrm{P}=$ 1.1e-08; PFS HR = 0.58, R = 7.6e-07), low CXCL9 expression was correlation to PFS (progression-free survival) and OS (poor overall survival). Furthermore, in OV and GC, CXCL9 was shown to have a close interaction with tumor-infiltrating immunity cells (B cells, CD4+ and CD8+ T cells, macrophages, neutrophils, and dendritic cells). CXCL9 expression, on the other hand, was shown to be closely related to several immune markers.

Conclusion: In OV and GC, CXCL9 mRNA level is strongly associated with prognosis and levels of penetration tumorinfiltrating immunity cell. The CXCL9 expression may also play a role in controlling TAMs (tumor-associated macrophages), DCs (Dendritic cells), CTLs (cytotoxic lymphocytes), and NK (natural killer) cells in OV and GC. CXCL9 may be seen as an independent marker that assesses the prognosis in OV and GC patients. Besides, CXCL9 expression level also can assess the immune cell subtypes of tumor microenvironment in OV and GC.

\section{Introduction}

Ovarian cancer and Gastric cancer are globally important diseases. Ovarian cancer is the second most common cause of allyear-round, after breast cancer, in women. Annually, there are over 239,000 new cases and 150,000 deaths. Patients in stages III or IV illness have a five-year average survival rate of $29 \%$. Since ovarian cancer is often asymptomatic at first, about $75 \%$ of women are already in advanced stages when they are diagnosed. It is estimated that one million new cases of gastric cancer arise per year, and the 5-year survival rate is below 25 percent[1-3]. Fortunately, immunotherapy has been emerging as a promising alternative treatment for some ovarian and gastric cancer patients. Some immunotherapy, for example, CTLA4 (cytotoxic T lymphocyte-associated antigen 4) therapies, PD-L1 (programmed death ligand-1), and PD-1 (programmed cell death protein-1) inhibitors have demonstrated an optimistic antitumor activity in ovarian cancer and gastric cancer. However, like other therapy, only a portion of patients benefit from immunotherapy[4-8]. More and more research found that the immune cell subtype in the tumor microenvironment affects immunotherapy efficacy in cancer treatment[9-13]. Therefore, the understanding of play role of immune cells in immunotherapy is urgently needed,and search new immunity treatment target.

Monokine induced by gamma interferon MIG/CXCL9 belongs to the CXC chemokine family[14]. The function of CXCL9 is to induce chemotaxis, facilitate the multiplication and differentiation of leukocytes, and cause extravasation of tissues. The CXCL9/CXCR3 receptor influences the migration, differentiation, and activation of immune cells [15]. Recently study observed that aberrant abnormal expression of CXCL9 in multiple cancer types. Besides, other research indicated that CXCL9 levels of gene expression associated with clinicopathological features of in multiple cancer types [16-20]. Thus, CXCL9 could have functions in tumorigenesis, growth, and metastases, and may function as the essential prognosis factor for tumors. However, the role of CXCL9 in ovarian and gastric cancers and possible molecular mechanisms has still not been explained so further exploration is necessary.

In this research, we have investigated the relationship between CXCL9 expression and prognosis in cancer patients. We also explored the impact of CXCL9 mRNA expression level on tumor-infiltrating immune cells based on a series of online public 
databases. Our result reveals CXCL9 plays a critical role in tumor immunity by regulating tumor-infiltrating immune cells in OV and GC.

\section{Materials And Methods}

\section{CXCL9 mRNA expression analysis}

In the Oncomine database, the CXCL9 mRNA expression level in different cancer types was identified[21]. The following are the threshold parameters: $p<0.001$, fold change $>2$.

\section{Clinical characteristics analysis}

The Kaplan-Meier plotter is an online database, including 54k genes and 14912 cancer samples, which can assess the different genes' effect on survival rates in 21 cancer types[22]. Using KM plotter (http://kmplot.com/analysis/) our team analyzed the association of CXCL9 expression to clinical characteristics in the breast, ovarian, lung, and gastric cancers. There were estimated 95\% confidence intervals for hazard ratios (HRs). Log-rank P-values were measured as well.

\section{TIMER analysis}

The Tumor IMmune Estimation Resource (TIMER) an online database that comprehensive analysis of tumor-infiltration cells[23]. TIMER analysis reveals the association between the CXCL9 expression and immune cell infiltration (9). TIMER gene modules were used to examine CXCL9 expression in multiple cancers and the association between CXCL 9 and the infiltrating level of various immune cells in the tumor microenvironment. Using TIMER correlation modules explored the interaction of CXCL9 expression with immune-related gene markers. Various immune cell molecular markers have been identified in previous research, for example, CCL2, CD68, and IL10 of TAM cell. The gene expression level has been shown by $\log 2$ TPM.

\section{GEPIA analysis}

Their genes that showed an obvious correlation in the TIMER study were further verified using the online database GEPIA[24]. GEPIA database offers their modules: Gene analysis, Custom data, and Cancer Type Analysis containing tumor and normal samples over18,000 sample from the GTEx projects and TCGA. The correlation of two genes was analyzed by the Spearman method.

\section{Results}

\section{CXCL9 Transcriptional Levels in a Variety of Tumors}

The Oncomine database analyzed the levels different of CXCL9 mRNA in various tumors and their normal tissues. It found out that CXCL9 expression was elevated in the following cancers: gastric, breast, bladder, cervical, head and neck, colorectal, kidney, leukemia, liver, lymphoma, and prostate (Figure 1A). using the TIMER database, we further assessed the CXCL9 expression difference in various cancer. Results show that CXCL9 expression was elevated in bladder urothelial carcinoma (BLCA), breast invasive carcinoma (BRCA), colon adenocarcinoma (COAD), cholangiocarcinoma (CHOL), head and neck cancer (HNSC), kidney renal clear cell carcinoma (KIRC), liver hepatocellular carcinoma (LIHC), esophageal carcinoma (ESCA), lung squamous (LUSC), lung adenocarcinoma (LUAD), rectum adenocarcinoma (READ), prostate adenocarcinoma (PRAD), uterine corpus endometrial carcinoma (UCEC), and stomach adenocarcinoma (STAD) than their normal tissues. instead, CXCL9 expression was low in thyroid carcinoma (THCA), kidney chromophobe (KICH), and kidney renal papillary (KIRP) than their normal tissues. (Figure 1B).

\section{The Prognostic value of CXCL9 in human cancers}


Next, Using KM plotter database to analyze whether the CXCL9 expression level impacts cancer patient's prognosis. The result shows that high CXCL9 level corresponded with better prognosis in OV (OS HR $=0.78, P=0.0017$; PFS HR $=0.85, P=$ $0.015), \mathrm{GC}$ patients $(\mathrm{OS} \mathrm{HR}=0.55, P=1.1 \mathrm{e}-08$; PFS HR $=0.58,95 \% \mathrm{Cl}=0.46-0.72, P=7.6 \mathrm{e}-07)$, Breast patients (OS HR = $0.88, P=0.0011$; RFS HR $=0.83, P=0.0039$; PPS HR $=0.74, P=0.022$, DMFS HR $=0.68, P=0.0011$ ), Lung patients (PFS HR $=0.84, P=0.015)$ and Liver patient (OS HR $=0.66, P=0.027$; PFS HR $=0.65, P=0.0083$ ) (Figure 2(A-H, G-L)). Yet, the CXCL9 expression was no correlation with OS in Lung cancer (Figure 2I). These results indicated that the CXCL9 expression impacts breast, liver, ovarian, and gastric cancer prognosis.

\section{CXCL9 expression impact the clinical characteristic of OV and GC patient}

Next, we are again using the Kaplan-Meier Plotter database to investigate whether CXCL9 expression affects the clinical characteristics of OV and GC patients. (Table 1). In stage 3 ovarian cancer patients, low CXCL9 expression show to be associated with a worse OS and PFS (OS HR $=0.73, P=0.0005$, PFS HR $=0.71, P=5.1 \mathrm{E}-05$ ). Overexpression CXCL9 was relationship with better OS and RFS of patients treated with Taxol, platin, and platin + Taxol chemotherapy. In stage 1-3 gastric cancer patients, high CXCL9 expression show to be associated with a better correlation with a better OS and PFS (OS $\mathrm{HR}=0.1, P=0.0066$, PFS HR $=0.14, P=0.027$; OS HR $=0.45, P=0.011$, PFS HR $=0.44, P=0.01$; OS HR $=0.52, P=2 \mathrm{e}-04$, PFS HR $=0.53, P=0.002$ ). Besides, the gastric patients at the N0-2 stage also showed an obvious correlation with CXCL9 expression. (OS HR $=0.19, P=0.011$, PFS HR $=0.2, P=0.014$; OS HR $=0.55, P=0.0061$, PFS HR $=0.53, P=0.0028 ;$ OS HR $=$ $0.36, P=0.0011$, PFS HR $=0.42, P=0.0035$ ) (Table 2 ). These findings show that CXCL9 expression has prognostic signification in OV and GC patients according to their clinical characteristics.

\section{CXCL9 expression impacts the immune cell infiltration from ovarian and gastric cancer.}

Cancer patients' survival times are affected by tumor-infiltrating lymphocytes. So we analyzed the correlation between the 39 cancer in TIMER and the CXCL9 expression level. The analyzed result indicated that CXCL9 expression associates with tumor purity in 27 types of cancer. besides, CXCL9 expression obviously association with the infiltration levels of immune cell. Such as $\mathrm{CD}^{+}$(26 types cancer), B cell (23 types cancer), CD $8^{+} T$ cells (32 types cancer), Macrophage cells (12 types cancer), Neutrophil cell (32 types cancer) and Dendritic cell (34 types cancer) (supplementary Figure 1). In OV and GC, high CXCL9 transcription was association to better prognosis and elevated immune cell infiltration levels. High CXCL9 expression was found to be obviously positive associated with the infiltration level of immune cell including $\mathrm{CD}^{+}(r=0.268, P=2.37 \mathrm{e}-$ 09), $B$ cells $(r=0.199, P=1.14 e-05)$, macrophages $(r=0.025, p=5.79 e-01), C D 8^{+} T$ cells $(r=0.401, P=5.15 e-20)$, neutrophils $(r=0.338, P=2.72 e-14)$, and DCs $(r=0.39, P=7.42 e-14)$, in OV tissues (Figure $3 A)$. Similar, in Gastric cancer has been found to be obviously correlation with better prognosis and elevated immune cell infiltration levels. Immune cell including $\mathrm{CD} 4^{+} \mathrm{T}$ cells $(r=0.131, P=1.20 E-02)$ CD $8^{+} T$ cells $(r=0.595, P=8.33 e-37)$, neutrophils $(r=0.521, P=3.60$ e-27), macrophages $(r=$ $0.179, P=5.34 e-04)$, and DCs $(r=0.551, P=8.53 e-31)$. Interestingly, Only B cells in GC had a negative association $(r=-0.209$, $P=5.44 \mathrm{e}-05$ ) (Figure $3 \mathrm{~B}$ ). The results clearly indicate that CXCL9 can recruit immune cells in the OV and GC microenvironment.

\section{CXCL9 Expression and Immune Marker Association Analyze}

The immune cell that infiltration level obviously correlation with the CXCL9 expression in OV and GC by KM plotter were further analyzed in TIMER and GEPIA database (Figure 4, Table3 and Table4). we found markers of immune cells in OV was to be strongly correlated with CXCL9 expression, including CD8+ T cell marker, CD8A $(r=0.739 ; P=2.78 e-44), C D 8 B(r=$ 0.585; $P=2.77 e-24)$, $T$ cell marker, $C D 3 D(R=0.83 ; P=1.56 e-64), C D 3 E(R=0.842 ; P=4.47 e-68), C D 2(r=0.852 ; P=1.73 e-$ 71), $B$ cell marker, CD79A ( $r=0.584 ; P=3.49 e-24)$, Monocyte marker, CD86 ( $r=0.512, P=4.56 e-18)$, TAM marker, CD68 $(r=$ 0.488; $P=2.73 e-16), M 2$ macrophage marker, MS4A4A ( $r=0.446, P=1.37 e-13)$ Neutrophils marker,CCR7 $(r=0.602, P=$ 6.66e-26), Nature killer cell marker, KIR2DL4 ( $r=0.482 ; P=7.41 \mathrm{e}-16)$, Dendritic cell marker, HLA-DPB1 $(r=0.507 ; P=1.12 \mathrm{e}-$ 17), HLA-DRA ( $r=0.447 ; P=1.49 e-15)$, HLA-DPA1 $(r=0.512, P=4.74 e-18)$ and CD11C $(r=0.421 ; P=4.09 e-12)$. Besides, we also found markers of immune cells in GC was to be strongly correlated with CXCL9 expression, including CD8+ T cell 
marker, CD8A ( $r=0.737 ; \mathrm{P}=4.56 \mathrm{e}-66), \mathrm{CD} 8 \mathrm{~B}(\mathrm{r}=0.604 ; \mathrm{P}=5.27 \mathrm{e}-39)$, $\mathrm{T}$ cell marker, CD3D $(r=0.707 ; \mathrm{P}=9.99 \mathrm{e}-59), \mathrm{CD} 3 \mathrm{E}(\mathrm{r}=$ 0.706; $P=1.82 e-58), C D 2(r=0.745 ; P=2.97 e-68)$, Monocyte marker, CD86 ( $r=0.643, P=1.27 e-45), C D 115(r=0.499 ; P=$ 2.83e-25), TAM marker, IL10 ( $r=0.404 ; P=2.57 e-16)$, M2 macrophage marker, CD163 ( $r=0.521 ; P=1.02 e-27), V S I G 4$ ( $r=$ 0.465; $P=1.10 \mathrm{e}-21)$, MS4A4A $(r=0.523 ; P=6.11 \mathrm{e}-28)$, Neutrophils marker, CD11b $(r=0.411 ; P=7.33 \mathrm{e}-17), \mathrm{CCR} 7(r=0.412$, $P=5.96 e-17)$, Nature killer cell marker, KIR2DL1 ( $r=0.412 ; P=5.96 e-17), K I R 2 D L 4(r=0.549 ; P=3.34 e-31), K I R 3 D L 1(r=$ 0.443; $P=1.08 \mathrm{e}-19)$, KIR3DL2 ( $r=0.437 ; P=4.10 \mathrm{e}-19)$,Dendritic cell marker, HLA-DPB1 ( $r=0.644 ; P=1.02 \mathrm{e}-45), \mathrm{HLA}-D Q B 1$ (0.508; $P=3.06 e-26)$, HLA-DRA $(r=0.637 ; p=1.90 e-44)$, HLA-DPA1 $(r=0.623 ; P=1.90 e-44)$ and CD11C $(r=0.497 ; P=5.24 e-$ 25) (Figure 4 and Table3). GEPIA analysis resulted have consistent with TIMER (Table 4). These results indicated that CXCL9 expression correlation with infiltration of immune cells in OV and GC.

\section{Discussion}

The role of CXCL9 in the tumorigenesis and metastasis of different tumors has always been controversial. For instance, Addison et al[15]. Found CXCL9 expression at high levels can inhibit tumor-derived angiogenesis, which inhibits NSCLC tumor development. Liber et al[25]. Found OV patients with elevated levels of CXCL9 had improved RFS. Chang et al[16]. Found a significant correlation between high cxcl9 expression and worse OS in OSCC (oral squamous cell carcinoma) patients. CXCL9 expression was associated with the prognosis of OV and GC in our research. In OV and GC, low CXCL9 expression was associated with a poor survival outcome. Besides, we discovered that the infiltration level of immune cells and different immunological markers are related to CXCL9 expression in OV and GS. Thus, our study suggested that CXCL9 potential plays a vital part in recruiting immune cells into tumor microenvironment progress, and it also can use as a prognosis biomarker. In the present research, analyzed CXCL9 mRNA expression level in multiple kinds of cancer by ONCOMINE and TIMER online database. levels of CXCL9 expression have been identified in multiple cancers and their normal tissues. Due to differences in data collection and processing methods between databases, these two databases' analyzed results differ slightly. Furthermore, KM plotter analysis shows increased CXCL9 expression level was shown to be associated with favorable prognosis in breast, ovarian, gastric, liver, and cancers (Fig. 3). In gastric patients, low CXCL9 expression with poor survival outcomes in stage 1-3, T2-3, and N0-2 disease. In ovarian patients, low CXCL9 expression was associated with worse survival outcomes in patients who underwent suboptimal debulking surgery and stage 3 disease. This finding strongly supports the notion that CXCL9 is a prognosis biomarker for ovarian and gastric cancers.

This study also discovered that the CXCL9 expression impacted the immune cell's infiltration level in OV and GC. There was a strong correlation between infiltration of CD8 + T cell, CD4 + T cell, Neutrophil, Dendritic cell, and B cell and CXCL9 expression in OV and GC. Moreover, the immune cells marker gene was positively correlated with CXCL9 expressions, such as TAM marker CD68, monocyte marker, CD86, and M2 macrophage marker, MS4A4A. This indicates that CXCL9 regulates the infiltration and activity of tumor-associated macrophages (TAMs) and activates cytotoxic lymphocytes (CTLs) and natural killer cells (NKs). These findings suggest that CXCL9 is essential for the recruitment and modulation of immune infiltrating cells in OV and GC.

\section{Conclusion}

In conclusion, our results suggest increasing CXCL9 expression associated with better prognosis and evaluating immune cell infiltration in OV and GC patients. Therefore,CXCL9 is a potential new independent biomarker evaluate for OV and GS patient prognosis and play a critical role regulation immune cell into the tumor microenvironment.

\section{Abbreviations}

CXCL9/MIG: Monokine Induced by Gamma Interferon (MIG); GC: Gastric cancer; OV: Ovarian Cancer; PD-1: programmed death-1; CTLA-4: Cytotoxic T - Lymphocyte Antigen 4; TIMER: Tumor Immune Estimation Resource; GEPIA: Gene Expression Profiling Interactive Analysis; OS: overall survival; DSS: disease-specific survival; PFS: progression-free survival; RFS, relapse- 
free survival; RFS: relapse-free survival; DMFS: distant metastasis-free survival; PPS: post progression survival; FP: first progression; TAMs: tumor-associated macrophages; NK cell: natural killer cells; DCs: Dendritic cells, CTLs: cytotoxic lymphocytes.

\section{Declarations}

\section{Acknowledgments}

Not applicable.

\section{Author Contributions}

SL, TW and YL conceived the project and wrote the manuscript. HZ, RS, DW, KZ, SX, and JZ participated in data analysis.

\section{Funding}

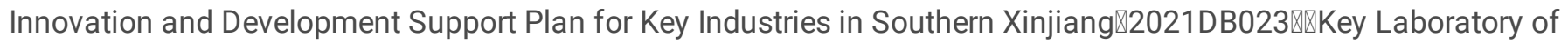

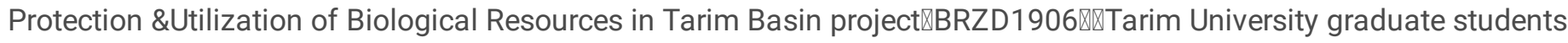
creation project $\triangle T$ DB SCX201905 $\$$ WNational natural science foundation of China (31560685).

\section{Availability of data and materials}

The authors declare that the data supporting the findings of this study are available within the article.

\section{Ethics approval and consent to participate Not applicable.}

Consent for publication Not applicable.

\section{Competing interests}

The authors declare that they have no competing interests.

\section{References}

1. de Martel, C., Georges, D., Bray, F., Ferlay, J. \& Clifford, G. M. Global burden of cancer attributable to infections in 2018: a worldwide incidence analysis. Lancet Glob Heal [Internet]. International Agency for Research on Cancer; 2020;8:e18090. Available from: http://dx.doi.org/10.1016/S2214-109X(19)30488-7

2. Bray, F. et al. Global cancer statistics 2018: GLOBOCAN estimates of incidence and mortality worldwide for 36 cancers in 185 countries. CA Cancer J Clin, 68, 394-424 (2018).

3. WHO \& Source, G. 2020. Globocan 2020 [Internet]. 2020;419:3-4. Available from: https://ascopost.com/news/december-2020/globocan-2020-database-provides-latest-global-data-on-cancer-burdencancer-deaths/\#: :text=Female breast cancer has now,with 685\%2C000 deaths in 2020.

4. Pawłowska, A. et al. Immunotherapies based on PD-1/PD-L1 pathway inhibitors in ovarian cancer treatment. Clin Exp Immunol, 195, 334-344 (2019).

5. Lampert, E. J. et al. Combination of PARP Inhibitor Olaparib, and PD-L1 Inhibitor Durvalumab, in Recurrent Ovarian Cancer: a Proof-of-Concept Phase II Study. Clin Cancer Res, 26, 4268-4279 (2020).

6. Färkkilä, A. et al. Immunogenomic profiling determines responses to combined PARP and PD-1 inhibition in ovarian cancer. Nat Commun [Internet]. Springer US; 2020;11:1-13. Available from: http://dx.doi.org/10.1038/s41467-020$15315-8$ 
7. Dai, C. et al. Implication of combined PD-L1/PD-1 blockade with cytokine-induced killer cells as a synergistic immunotherapy for gastrointestinal cancer. Oncotarget [Internet]. Impact Journals LLC, 7, 10332-10344 (2016). Available from: /pmc/articles/PMC4891123/

8. Muro, K. et al. Pembrolizumab for patients with PD-L1-positive advanced gastric cancer (KEYNOTE-012): a multicentre, open-label, phase 1b trial. Lancet Oncol [Internet]. Elsevier Ltd; 2016;17:717-26. Available from: http://dx.doi.org/10.1016/S1470-2045(16)00175-3

9. Mockler, M. B., Conroy, M. J. \& Lysaght, J. Targeting T cell immunometabolism for cancer immunotherapy; understanding the impact of the tumor microenvironment [Internet]. Front. Oncol. Frontiers Research Foundation; 2014 [cited 2021 Mar 15]. Available from: /pmc/articles/PMC4032940/

10. Baginska, J. et al. The critical role of the tumor microenvironment in shaping natural killer cell-mediated anti-tumor immunity [Internet]. Front. Immunol. Frontiers Media SA; 2013 [cited 2021 Mar 15]. Available from: /pmc/articles/PMC3872331/

11. Tekpli, X. et al. An independent poor-prognosis subtype of breast cancer defined by a distinct tumor immune microenvironment.Nat Commun [Internet]. Nature Research; 2019 [cited 2021 Mar 15];10. Available from: /pmc/articles/PMC6890706/

12. Matias, B. F. et al. Influence of immunotherapy with autologous dendritic cells on innate and adaptive immune response in cancer. Clin Med Insights Oncol [Internet]7165-72(Libertas Academica Ltd., 2013). Available from: /pmc/articles/PMC3733716/

13. Jäger, D., Jäger, E. \& Knuth, A. Immune responses to tumour antigens: Implications for antigen specific immunotherapy of cancer [Internet]. J. Clin. Pathol. BMJ Publishing Group; 2001 [cited 2021 Mar 15]. p.669-74. Available from: www.jclinpath.com

14. Lee, H. H. \& Farber, J. M. Localization of the gene for the human MIG cytokine on chromosome $4 q 21$ adjacent to INP10 reveals a chemokine "mini-cluster"i i ¿ $\iota^{1 / 2}$. Cytogenet Genome Res [Internet]. Cytogenet Cell Genet; 1996 [cited 2021 Feb 23];74:255-8. Available from: https://pubmed.ncbi.nlm.nih.gov/8976378/

15. Addison, C. L. et al. The CXC chemokine, monokine induced by interferon- $y$, inhibits non-small cell lung carcinoma tumor growth and metastasis. Hum Gene Ther, 11, 247-261 (2000).

16. Chang, K. P. et al. Serum levels of chemokine (C-X-C motif) ligand 9 (CXCL9) are associated with tumor progression and treatment outcome in patients with oral cavity squamous cell carcinoma. Oral Oncol [Internet]. Elsevier Ltd; 2013;49:802-7. Available from: http://dx.doi.org/10.1016/j.oraloncology.2013.05.006

17. Zhang, Y. et al. CXCL9 as a Prognostic Inflammatory Marker in Early-Stage Lung Adenocarcinoma Patients. Front Oncol, $10,1-7$ (2020).

18. Wu, Z. et al. The chemokine CXCL9 expression is associated with better prognosis for colorectal carcinoma patients. Biomed Pharmacother [Internet]. Elsevier Masson SAS; 2016;78:8-13. Available from: http://dx.doi.org/10.1016/j.biopha.2015.12.021

19. Hsin, L. J. et al. Serum CXCL9 levels are associated with tumor progression and treatment outcome in patients with nasopharyngeal carcinoma. PLoS One, 8, 1-8 (2013).

20. Li, Y. et al. Analysis of the Prognosis and Therapeutic Value of the CXC Chemokine Family in Head and Neck Squamous Cell Carcinoma. Front Oncol, 10, 1-13 (2021).

21. Rhodes, D. R. et al. ONCOMINE: A Cancer Microarray Database and Integrated Data-Mining Platform. Neoplasia, 6, 1-6 (2004).

22. Nagy, Ã., Lánczky, A., Menyhárt, O. \& Gyorffy, B. Validation of miRNA prognostic power in hepatocellular carcinoma using expression data of independent datasets.Sci Rep [Internet]. NaturePublishing Group; 2018 [cited 2021 Mar 15];8. Available from: /pmc/articles/PMC6003936/

23. Li, T. et al. TIMER: A web server for comprehensive analysis of tumor-infiltrating immune cells. Cancer Res, 77, e108-10 (2017). 
24. Tang, Z. et al. GEPIA: A web server for cancer and normal gene expression profiling and interactive analyses. Nucleic Acids Res, 45, W98-102 (2017).

25. Lieber, S. et al. Prognosis of ovarian cancer is associated with effector memory CD $8+T$ cell accumulation in ascites, CXCL9 levels and activation-triggered signal transduction in T cells.Oncoimmunology. 2018;7.

\section{Tables}

Table1. Correlation of CXCL9 mRNA expression and clinical prognosis in ovarian cancer 
Variables of ovarian cancer

\section{$N$}

\section{Histology}

Endometroid

Serous

Serous

\section{Stage}

1

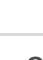

2

3

4

4176

\section{Grade}

1
2
3

\section{TP53 mutation}

Mutated

Wild type

Debulk

Optimal

Suboptimal

\section{Chemotherapy}

contains platin

contains Taxol

contains Taxol+platin
Overall survival $(\mathrm{N}=1656)$

Progression-free survival ( $\mathrm{N}=1436)$

Hazard P-value

ratio

37

$2.76(0.46-$

0.25

16.52)

1207

$0.76(0.63-$
$0.91)$

$0.91)$

$N \quad$ Hazard ratio

51

$2.94(1.16-$
$7.46)$

0.018

0.0035

110

104

$0.77(0.66-$

$0.89)$

0.0005

$P$ value
2.81 (0.88-

8.94)

61

1044

176

$\begin{array}{ll}56 & 0.43(0.16- \\ & 1.17)\end{array}$

324

1015

1.13)

$0.66(0.55-$

$0.81)$

0.44 (0.15-

1.32)

$0.73(0.61-$
$0.87)$

$0.81(0.56-$

1.19)
$0.82(0.59-$

0.068

0.13

0.0005

0.0005

0.28

0.09

0.22

0.000031

$0.000031 \quad 837$

837

$0.71(0.6$
$0.85)$

$\begin{array}{lll}37 & \begin{array}{l}4.05(1.31- \\ 12.58)\end{array} & 0.009 \\ & & \\ 256 & \begin{array}{l}1.33(0.99- \\ 1.8)\end{array} & 0.059 \\ & & \end{array}$

$.47(0.96$

2.26) 
Variables of gastric cancer Overall survival $(\mathrm{N}=875 \square$

Progression-free survival $(\mathrm{N}=640)$

$\boldsymbol{N}$ Hazard ratio $P$-value $N$ Hazard ratio $P$-value

Gender

\begin{tabular}{lllllll} 
Female & 236 & $0.46(0.3-0.72)$ & 0.0005 & 201 & $0.53(0.33-0.86)$ & 0.009 \\
\hline Male & 544 & $0.59(0.47-0.74)$ & $4.00 \mathrm{E}-06$ & 437 & $0.53(0.4-0.68)$ & $1.20 \mathrm{E}-06$
\end{tabular}

Stage

\begin{tabular}{lllllll}
\hline 1 & 67 & $0.1(0.01-0.77)$ & 0.0066 & 60 & $0.14(0.02-1.08)$ & 0.027 \\
\hline 2 & 140 & $0.45(0.24-0.84)$ & 0.011 & 131 & $0.44(0.23-0.84)$ & 0.01 \\
\hline 3 & 305 & $0.52(0.36-0.74)$ & $2.00 \mathrm{E}-04$ & 186 & $0.53(0.35-0.8)$ & 0.002 \\
\hline 4 & 148 & $0.52(0.32-0.83)$ & 0.0054 & 141 & $0.65(0.41-1.04)$ & 0.069
\end{tabular}

\section{Stage T}

$\begin{array}{lllllll}2 & 241 & 0.56(0.33-0.95) & 0.029 & 239 & 0.42(0.24-0.76) & 0.0032 \\ 3 & 204 & 0.54(0.36-0.79) & 0.0016 & 204 & 0.61(0.42-0.89) & 0.0089 \\ 4 & 38 & 0.67(0.26-1.71) & 0.4 & 39 & 1.67(0.63-4.42) & 0.3\end{array}$

\section{Stage $\mathbf{N}$}

\begin{tabular}{lllllll}
\hline 0 & 74 & $0.19(0.04-0.8)$ & 0.011 & 72 & $0.2(0.05-0.83)$ & 0.014 \\
\hline 1 & 225 & $0.55(0.36-0.85)$ & 0.0061 & 222 & $0.53(0.35-0.81)$ & 0.0028 \\
\hline 2 & 121 & $0.36(0.19-0.68)$ & 0.0011 & 125 & $0.42(0.23-0.77)$ & 0.0035 \\
\hline 3 & 76 & $0.61(0.32-1.16)$ & 0.13 & 76 & $0.65(0.34-1.24)$ & 0.19
\end{tabular}

\section{Stage M}

$\begin{array}{lllllll}0 & 444 & 0.53(0.39-0.71) & 2.40 \mathrm{E}-05 & 443 & 0.56(0.42-0.75) & 6.60 \mathrm{E}-05 \\ 1 & 56 & 0.58(0.32-1.04) & 0.065 & 56 & 0.73(0.37-1.44) & 0.36\end{array}$

\section{HER2 status}

\begin{tabular}{lllllll} 
negative & 532 & $0.43(0.32-0.59)$ & $3.90 \mathrm{E}-08$ & 408 & $0.49(0.36-0.66)$ & $2.80 \mathrm{E}-06$ \\
\hline positive & 343 & $0.74(0.54-1.01)$ & 0.058 & 232 & $1.36(0.98-1.89)$ & 0.063
\end{tabular}

\section{Lauren classification}

$\begin{array}{lllllll}\text { Intestinal } & 320 & 0.6(0.42-0.84) & 0.0026 & 263 & 0.5(0.33-0.76) & 0.0008 \\ \text { Diffuse } & 241 & 0.61(0.41-0.91) & 0.015 & 231 & 0.6(0.39-0.92) & 1.80 \mathrm{E}-02\end{array}$

Differentiation

\begin{tabular}{lllllll} 
poorly & 165 & $0.54(0.35-0.84)$ & 0.0051 & 121 & $1.78(0.99-3.2)$ & 0.05 \\
\hline moderately & 67 & $1.88(0.98-3.6)$ & 0.053 & 67 & $1.83(0.98-3.42)$ & 0.054
\end{tabular}

Table3. Correlation analysis between CXCL9 and relate genes and markers of immune cells in TIMER. 
OV

Purity

Description

CD8+ T cell

T cell
(general)

\section{Cor}

Gene
markers

CD8A

CD8B

CD3D
CD3E

CD2

B cell

CD19

CD79A

Monocyte

CD86

CD115

(CSF1R)

TAM

CCL

CD68

IL10

M1 INOS

macrophage (NOS2)

\begin{tabular}{lll} 
IRF5 & 0.11 & 0.083 \\
COX2 & 0.003 & 0.963 \\
(PTGS2) & & \\
\hline
\end{tabular}

M2

macrophage

CD163

0.358

$\star \star \star *$

0.278

VSIG4

MS4A4A

0.446
Neutrophils

CD11b

(ITGAM)

CD66b

(CEACAM8)

CCR7

Natural killer KIR2DL1

cell
STAD

\section{Purity}

Cor $P$

0.737 ***

$0.604 \quad \star \star \star$

0.707 ***

0.706 ***

0.745 ***

$0.885 * \star *$

0.293 ***

0.679

$0.677 * \star \star \star$

0.518 ***

$0.461 \quad * \star *$

0.632

0.366 ***

$-0.014 \quad 0.803$

0.219 **

0.157 **

0.536

$\star \star \star$

0.491

0.6

0.532

$-0.104 \quad 0.065$

$-0.093 \quad 0.145$

0.602

0.671

0.228 ***

$0.124 \quad 0.051$

KIR2DL3

$0.139 \quad 0.028$

0.203

$0.544 \quad \star \star *$

KIR2DL4

0.482 ***

KIR3DL1

0.259

KIR3DL2

0.188 *

KIR3DL3

$0.121 \quad 0.057$

0.328 ***

0.375 ***

$0.643 \quad * * *$

$0.499 * \star \star$

$0.229 * \star \star *$

0.35

0.40

$0.065 \quad 0.209$

0.215 ***

$\begin{array}{ll}-0.089 & 0.084\end{array}$

0.521

$\star \star \star$

0.465

***

0.523

$\star \star \star$

0.411

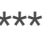

$\begin{array}{ll}-0.014 & 0.791\end{array}$

$0.412 \quad * \star \star$

0.367 ***

0.44

0.39

$-0.002$

0.791

$0.419 \quad * \star *$




\begin{tabular}{|c|c|c|c|c|c|c|c|c|c|}
\hline & KIR2DS4 & 0.111 & 0.080 & 0.203 & $\star \star$ & 0.362 & $\star \star \star$ & 0.376 & $\star \star \star *$ \\
\hline \multirow{7}{*}{$\begin{array}{l}\text { Dendritic } \\
\text { cell }\end{array}$} & HLA-DPB1 & 0.507 & $\star \star *$ & 0.641 & $\star \star \star *$ & 0.644 & $\star \star \star$ & 0.659 & $\star \star \star$ \\
\hline & HLA-DQB1 & 0.333 & $\star \star *$ & 0.452 & $\star \star \star$ & 0.508 & $\star \star \star *$ & 0.535 & $\star \star \star *$ \\
\hline & HLA-DRA & 0.447 & $\star \star \star *$ & 0.604 & $\star \star \star *$ & 0.637 & $\star \star \star$ & 0.691 & $\star \star \star *$ \\
\hline & HLA-DPA1 & 0.512 & $\star \star \star *$ & 0.638 & $\star \star \star$ & 0.623 & $\star \star \star *$ & 0.639 & $\star \star *$ \\
\hline & $\begin{array}{l}\text { BDCA-1 } \\
\text { (CD1C) }\end{array}$ & 0.219 & ** & 0.397 & $\star \star \star *$ & 0.18 & $\star \star \star \star$ & 0.212 & $\star \star \star *$ \\
\hline & $\begin{array}{l}\text { BDCA-4 } \\
\text { (NRP1) }\end{array}$ & 0.105 & 0.098 & 0.303 & $\star \star \star *$ & 0.274 & $\star \star \star$ & 0.288 & $\star * *$ \\
\hline & $\begin{array}{l}\text { CD11c } \\
\text { (ITGAX) }\end{array}$ & 0.421 & $\star \star * *$ & 0.57 & $\star \star *$ & 0.497 & $\star \star \star *$ & 0.506 & $\star \star *$ \\
\hline
\end{tabular}

Table4. Correlation analysis between CXCL9 and relate genes and markers of monocyte and macrophages in GEPIA

\begin{tabular}{|c|c|c|c|c|c|}
\hline \multirow[t]{3}{*}{ Description } & \multirow[t]{3}{*}{ Gene markers } & \multicolumn{2}{|l|}{ ov } & \multicolumn{2}{|l|}{ STAD } \\
\hline & & \multicolumn{2}{|c|}{ Tumor } & \multicolumn{2}{|c|}{ Tumor } \\
\hline & & $\mathbf{R}$ & $\mathbf{P}$ & $\mathbf{R}$ & $\mathbf{P}$ \\
\hline \multirow[t]{2}{*}{ Monocyte } & CD86 & 0.7 & $\star \star \star ~$ & 0.64 & $\star \star \star$ \\
\hline & CD115 (CSF1R) & 0.55 & $\star \star \star$ & 0.48 & $\star \star \star$ \\
\hline \multirow[t]{3}{*}{ TAM } & CD68 & 0.63 & $\star * *$ & 0.33 & $\star * *$ \\
\hline & CCL2 & 0.47 & 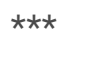 & 0.2 & $\star \star \star$ \\
\hline & IL10 & 0.45 & $\star \star \star$ & 0.4 & $\star \star \star *$ \\
\hline \multirow[t]{3}{*}{ M1 Macrophage } & INOS (NOS2) & 0.11 & 0.08 & 0.077 & 0.12 \\
\hline & IRF5 & 0.27 & $\star \star \star$ & 0.2 & $\star \star \star *$ \\
\hline & COX2 (PTGS2) & 0.22 & $\star \star *$ & -0.04 & 0.42 \\
\hline \multirow[t]{3}{*}{ M2 Macrophage } & CD163 & 0.57 & $\star \star \star \star$ & 0.53 & 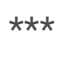 \\
\hline & VSIG4 & 0.53 & $\star \star \star *$ & 0.47 & 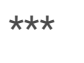 \\
\hline & MS4A4A & 0.63 & $\star * \star$ & 0.52 & $* * *$ \\
\hline
\end{tabular}

\section{Figures}



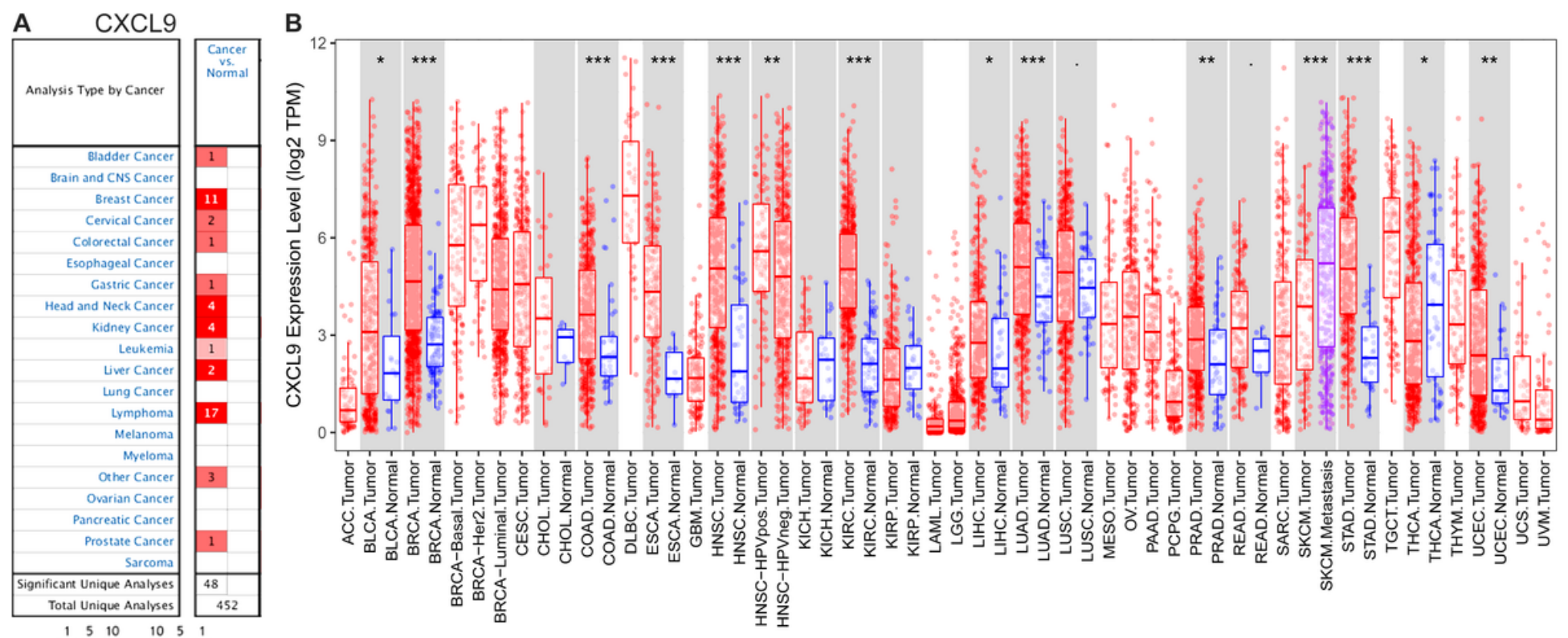

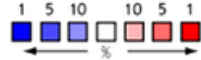

\section{Figure 1}

CXCL9 expression levels in different types of cancer. (A) CXCL9 expression level in data sets of different cancer types compared with normal tissues from Oncomine database. (B) CXCL9 expression levels in different types of cancer from the TCGA database in TIMER. Note: ${ }^{*} \mathrm{P}<0.05,{ }^{*} \mathrm{P}<0.01$, ${ }^{* \star} \mathrm{P}<0.001$. 
A Ovrian cancer,os

203915-at

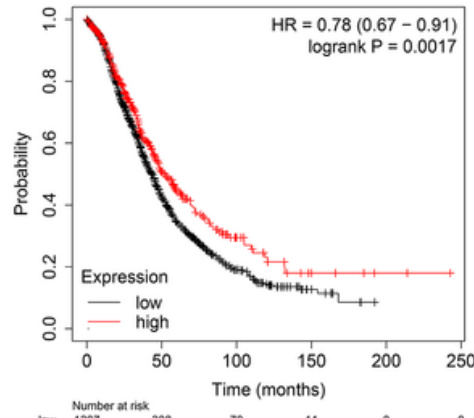

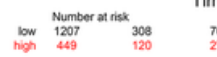

E Breast cancer,OS

203915-at

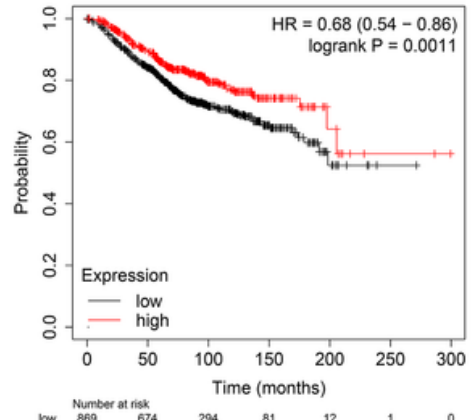

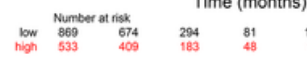

I Lung cancer,OS

203915-at

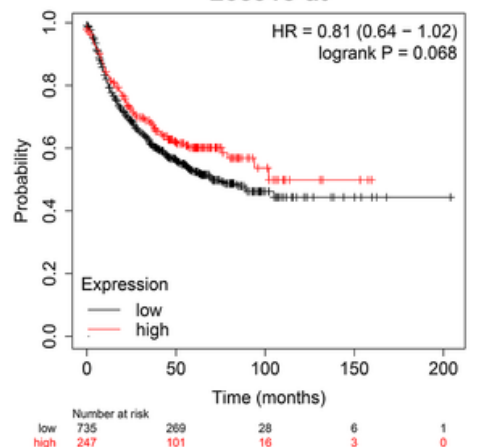

B Ovrian cancer,PFS

203915-at

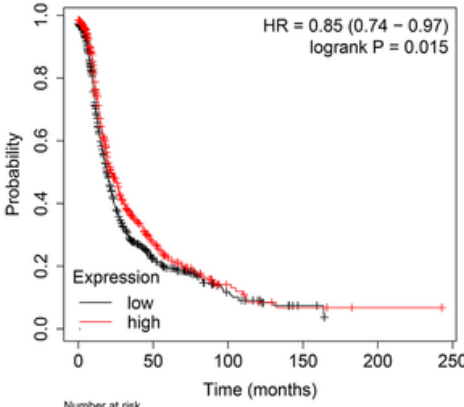

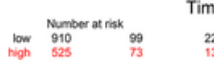

F Breast cancer,RFS 203915-at

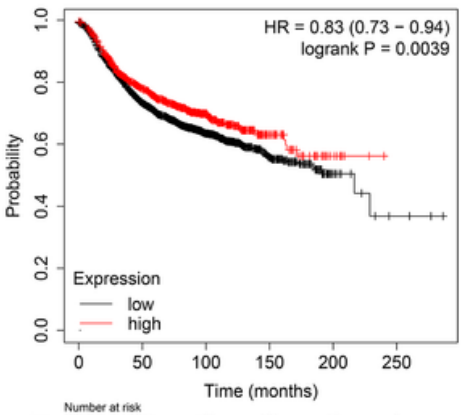

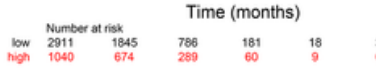

G Lung cancer,PFS

203915-at

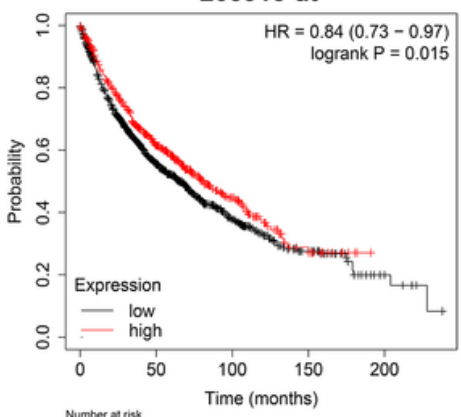

C Gastric cancer, OS

203915-at

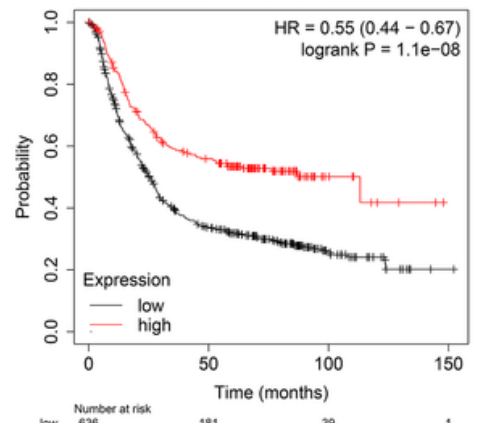

G Breast cancer,PPS

203915-at

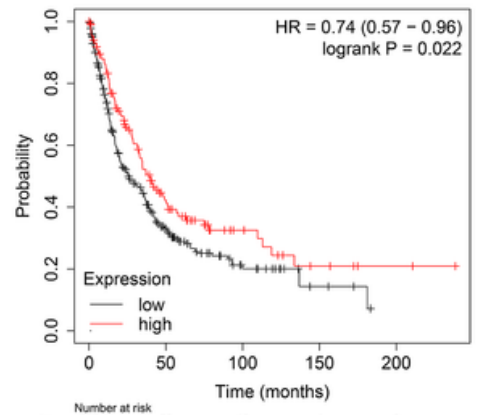

$\underset{\substack{\text { how } \\ \text { high }}}{\substack{\text { Number at iak } \\ 137}}$

K Liver cancer,OS

4283

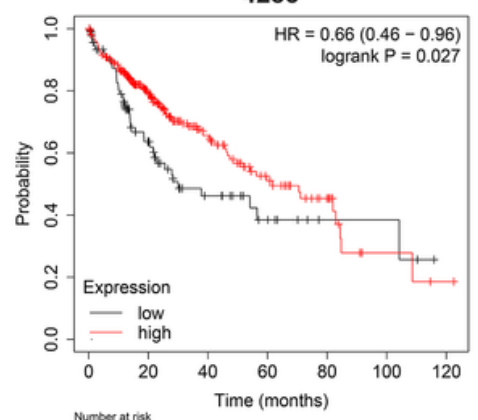

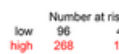

D Gastric cancer, PFS 203915-at

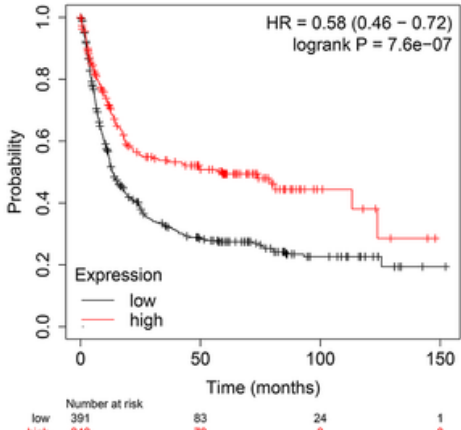

H Breast cancer,DMFS 203915-at

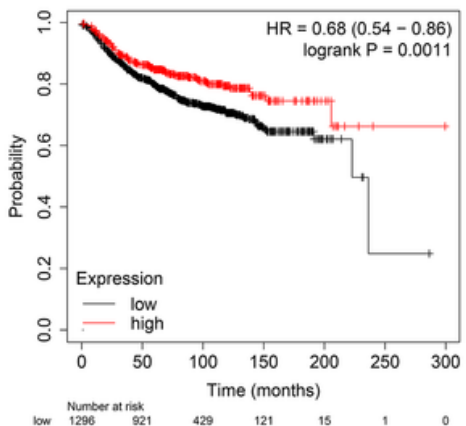

L Liver cancer,PFS 4283

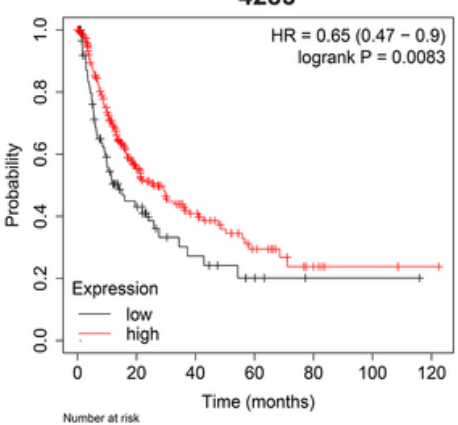

\section{Figure 2}

Kaplan-Meier survival curve analysis of the prognostic significance of high and low expression of CXCL9 in different types of human cancers using the Kaplan-Meier plotter database. (A-B) Kaplan-Meier survival analysis of CXCL9 in OS and PFS in ovarian cancer $(n=1656, n=1435)$. (C-D) Kaplan-Meier survival analysis of CXCL9 in OS and PFS in gastric cancer $(n=875$, $n=636)$. $(E-H)$ Kaplan-Meier survival analysis of CXCL9 in OS, RFS, PPS and DMFS in breast cancer $(n=1402, n=3951, N=$ $414, N=1803)$. (I-G) Kaplan-Meier survival analysis of CXCL9 in OS and PFS in lung cancer $(n=982, n=1925)$. $(K-L)$ Kaplan-Meier survival analysis of CXCL9 in OS and PFS in liver cancer $(n=364, n=370)$. 


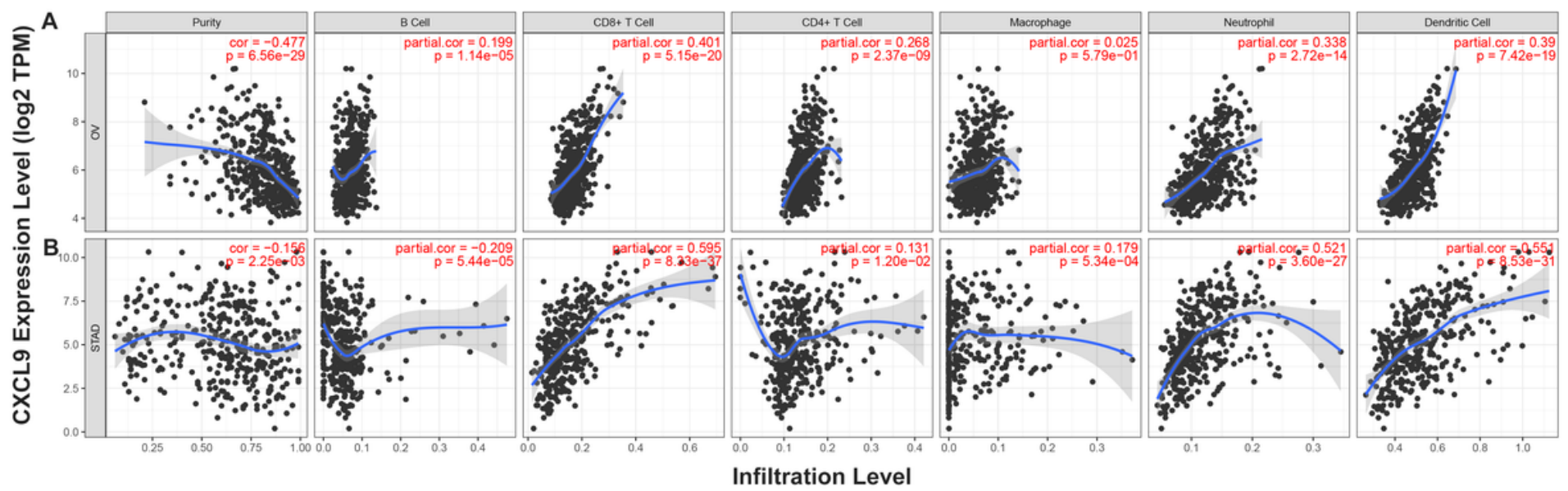

Figure 3

Correlation of CXCL9 expression with immune infiltration level in OV (ovarian serous cystadenocarcinoma) and STAD (stomach adenocarcinoma). (A) CXCL9 expression was significantly negatively related to tumor purity and had significant positive correlations with the levels of infiltrating B cells, CD8+ T cells, CD4+ T cells, neutrophils, and dendritic cells in OV. CXCL9 expression showed no correlation with macrophage infiltration in OV. (B) CXCL9 expression was significantly negatively related to tumor purity and had significant positive correlations with the levels of infiltrating CD8+ T cells, CD4+ $T$ cells, macrophages, neutrophils, and dendritic cells but had significant negative correlations with the levels of infiltrating $B$ cells in STAD.
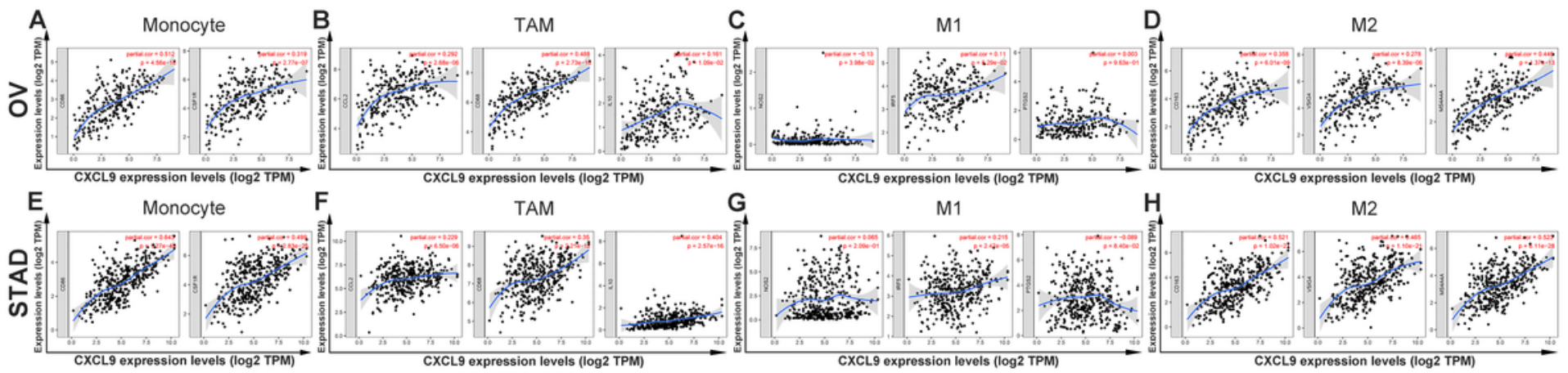

Figure 4

The correlation between the expression level of CXCL9 and immune marker genes in STAD and OV. Markers include CD86 and CSF1R of monocytes; CCL2, CD68, and IL10 of TAMs; NOS2, IRF5, and PTGS2 of M1 macrophages; and CD163, VSIG4, and MS4A4A of M2 macrophages. (A-D) Scatterplots of correlations between CXCL9 expression and gene markers of monocytes (A), TAMs (B), and M1 (C) and M2 macrophages (D) in OV. (E-H) Scatterplots of correlation between CXCL9 expression and the expression of gene markers of monocytes (E), TAMs (F), and M1 (G) and M2 macrophages (H) in STAD.

\section{Supplementary Files}

This is a list of supplementary files associated with this preprint. Click to download.

- supplementary.docx 